\title{
Horticultura comunitária e construção de sistemas socioecológicos sustentáveis
} Community horticulture and construction of sustainable socio-ecological systems

\author{
Alessandra Pavesi | UFSCar ${ }^{1}$ \\ Denise de Freitas | USP² \\ Bárbara Pacheco Lopes | UFSCar
}

\section{Resumo}

Neste trabalho, reafirma-se a importância da horticultura comunitária como ferramenta para a aprendizagem de conhecimentos e competências científicas e políticas necessárias para a construção de Sistemas Socioecológicos (SSE) sustentáveis e resilientes, ou seja, aptos para responder a perturbações e renovar-se mediante o fortalecimento de sua capacidade adaptativa. Para tanto, exploram-se conceitos, metáforas e teorias procedentes da (Agro)Ecologia e da Educação, a começar pela ideia de SSE, que nasce na confluência de domínios disciplinares tradicionalmente separados. Em seguida, as hortas comunitárias e escolares são reenquadradas nas perspectivas da aprendizagem contextualizada e da Teoria da Atividade, para explicitar suas potencialidades para a educação ambiental, a aprendizagem significativa das ciências e, sobretudo, para religar os mundos tradicionalmente separados dos seres humanos e da natureza.

Palavras-chave: hortas comunitárias, sistemas socioecológicos, sustentabilidade, resiliência, adaptabilidade, aprendizagem

\begin{abstract}
This paper seeks to reaffirm the importance of community horticulture as a tool for learning scientific and political contents and skills necessary for the construction of sustainable and resilient Socioecological Systems (SES), i.e., able to respond to disruptions and renew themself by strengthening their adaptive capacity. To this end, we shall explore whether concepts, metaphors and theories from (Agro)Ecology and Education, starting with the idea of SES, which is born at the confluence of traditionally separate disciplinary domains. Then, community and school gardens will be reframed from the point of view of contextualized learning and Activity Theory, to clarify their potential for meaningful learning of Sciences and, especially, to re-bind the traditionally separate worlds of humans and nature.
\end{abstract}

Key words: community gardens, socioecological systems, sustainability, resilience, adaptability, learning.

\footnotetext{
${ }^{1}$ Com apoio da CAPES - PNPD Institucional

${ }^{2}$ Com apoio parcial do CNPq
} 


\section{Introdução}

O cultivo de hortas firmou-se como prática comum nas escolas brasileiras, incentivada também por projetos e programas nacionais e municipais. Embora não se conte com dados conclusivos sobre o número de hortas escolares em funcionamento, uma rápida incursão na WEB permite conhecer inúmeras iniciativas em suas propostas educativas e resultados. Um exemplo representativo por abordar pressupostos teóricos e aspectos práticos da horticultura escolar é o Projeto Educando com a Horta Escolar ${ }^{3}$, realizado pelo Fundo Nacional de Desenvolvimento da Educação (FNDE) do Ministério da Educação (MEC) em parceria com a Organização das Nações Unidas para a Agricultura e Alimentação (FAO), que coloca a ênfase na importância da horticultura para a aprendizagem significativa e a educação alimentar.

Neste nosso exercício, reafirma-se a importância da horticultura escolar, particularmente no que se refere a uma função tão específica quanto compreensiva das múltiplas dimensões da sustentabilidade ${ }^{4}$, que consiste na construção da resiliência e capacidade adaptativa de comunidades e sistemas socioecológicos (SSE) frente a perturbações e crises. O próprio conceito de SSE contém uma promessa de superação, por implicar possibilidades de integração (e colaboração) entre campos disciplinares tradicionalmente separados, com a perspectiva de produzir efeitos na compreensão e gestão de sistemas complexos formados pelas componentes natural e social (seus atores e instituições).

Assim, nas seções seguintes, exploram-se algumas propriedades dos SSE, tais como a complexidade, a adaptabilidade e a resiliência, para, em seguida, discutir a importância da educação e da aprendizagem na construção de SSE sustentáveis e resilientes. Por fim, as hortas escolares são reenquadradas nas perspectivas da aprendizagem contextualizada e da Teoria da Atividade, como potenciais laboratórios para aprendizagens múltiplas: científicas, cívicas e políticas necessárias para religar os mundos tradicionalmente separados dos seres humanos e da natureza.

\section{Sustentabilidade e resiliência em Sistemas Socioecológicos (SSE)}

A sustentabilidade de SSE realiza-se no encontro de três qualidades inerentes a suas estruturas e processos: viabilidade econômica, integridade ecológica, justiça e coesão social. Esta intersecção admite inúmeras soluções concretas, que dependem não só da diversidade dos contextos ecológicos, mas, sobretudo, do desenvolvimento de novos modelos de integração entre sistemas sociais (suas formações e instituições) e naturais. Tal desafio

\footnotetext{
${ }^{3}$ O projeto explana-se em três cadernos intitulados: 1. A horta escolar dinamizando o currículo da escola, 2. Orientações para implantação e implementação da horta escolar, 3. Alimentação e nutrição - caminhos para uma vida saudável.

${ }^{4}$ Sustentabilidade social, que se fundamenta na equidade; sustentabilidade econômica, que requer a alocação e o gerenciamento mais eficiente (do ponto de vista macrossocial) dos recursos; sustentabilidade ecológica, que deriva da proteção dos sistemas de sustentação da vida; sustentabilidade espacial, que demanda uma configuração urbano-rural mais equilibrada; sustentabilidade cultural, que implica a busca das raízes endógenas de processos de modernização (Sachs, 1994).
} 
pressupõe, para Westley et al. (2002), a compreensão das características essenciais que determinam a dinâmica de ambos os sistemas.

No caso dos sistemas sociais, a principal consistiria na capacidade que os seres humanos têm de inventar e reinventar, por meio da comunicação simbólica, uma ordem significativa da qual se imergem e atuam como se fosse real. Esta propriedade permite-lhes transcender as dimensões mais críticas para a auto-organização dos ecossistemas: o espaço e o tempo. Na sociedade urbano-industrial, de fato, já não se marca o tempo com base em fenômenos físicos e culturais locais, e o próprio "local" é sempre mais atravessado e vulnerável a fatores distantes, em virtude da criação de sistemas simbólicos globalizantes ( $p$. ex. monetário), da desterritorialização das organizações sociais e do consequente descomprometimento com o local geográfico.

Ainda que a reflexividade (Beck et al, 1997), entendida como movimento de contestação das tradições e de renovação cultural, tenha aberto novas perspectivas para o equacionamento dos problemas inerentes à sociedade globalizada, segundo Westley et al. (op.cit.) está longe de compreender a dinâmica de sistemas complexos, nem dá conta de variáveis sujeitas a mudanças muito lentas. Também por isto, tomam-se decisões que, embora inicialmente possam parecer lógicas e eficazes, em longo prazo provocam efeitos ambientais catastróficos.

Talvez, a Revolução Verde represente um dos momentos culminantes desta tendência, manifesta, sobretudo, nas inovações tecnológicas pautadas na mercantilização da natureza. Em geral, estas são produzidas de acordo com uma lógica linear, que se contrapõe aos processos cíclicos naturais; intervêm em sistemas complexos dos quais se consideram poucas variáveis; contemplam uma única escala dos problemas que se deseja resolver e, desta forma, criam novos problemas em outras escalas, no tempo e no espaço. Por exemplo, a fabricação de fertilizantes a partir da fixação sintética de nitrogênio com o objetivo de incrementar a produtividade agrícola causou impactos ambientais subestimados, tais como a acumulação em níveis tóxicos de nitrato nos aquíferos, a emissão de gases de efeito estufa, a eutrofização e a proliferação de algas nos corpos d'água, a perda da biodiversidade, a crescente dependência de insumos industriais e a perda da soberania alimentar e genética.

Até mesmo as chamadas tecnologias ambientais, que têm como propósito a gestão dos limites biofísicos para o crescimento econômico (Sachs, 1995) e se remetem aos conceitos de resistência, estabilidade, controle e eficiência, não fogem às limitações apontadas anteriormente. Antes, sua fixidez (comparada por Westley et al. a uma viga imersa em bloco de concreto) tem contribuído para minar a resiliência dos SSE, entendida como capacidade de manter a própria identidade e reorganizar-se para buscar novos estados de equilíbrio, a partir do desenvolvimento de modelos endógenos de prosperidade. A resiliência depende, de fato, da adaptabilidade, informada pela imprevisibilidade e indeterminação, e sua busca evoca a dinâmica de ecossistemas, que "resolvem seus problemas" mediante um arranjo imbricado de mecanismos de resposta operantes em diferentes escalas temporais (o qual traz à mente os movimentos de uma árvore no vento).

O ciclo adaptativo representa a ferramenta metafórica ${ }^{5}$ que melhor expressa a noção de resiliência como processo de auto-organização dos sistemas, em consequência de

\footnotetext{
${ }^{5}$ Por metáforas entendem-se imagens que estimulam a criação de conceitos técnicos ou os representam mediante discursos informais ou não técnicos. Como atos da imaginação, essas figuras de linguagem tornam possível visualizar analogias que
} 
mudanças imprevistas (Holling e Gunderson, 2002). Composto por dois momentos, ilustra a maneira pela qual variáveis interagem para se fortalecer mutuamente e construir a organização de um sistema, e o modo pelo qual a dependência dessa organização torna o sistema mais vulnerável a perturbações, que eventualmente provocam a sua destruição abrindo oportunidades para a reorganização. Ensina, desta forma, que as crises podem pavimentar o caminho para rupturas e a transição para diferentes estados de equilíbrio (Sterling, 2010). Contudo, sua realização plena depende da capacidade do sistema de lidar com a incerteza e a mudança, cultivar a diversidade necessária para a renovação, de utilizar diversas tradições de conhecimento para aprender e criar oportunidades para autoorganizar-se (Plummer, 2010).

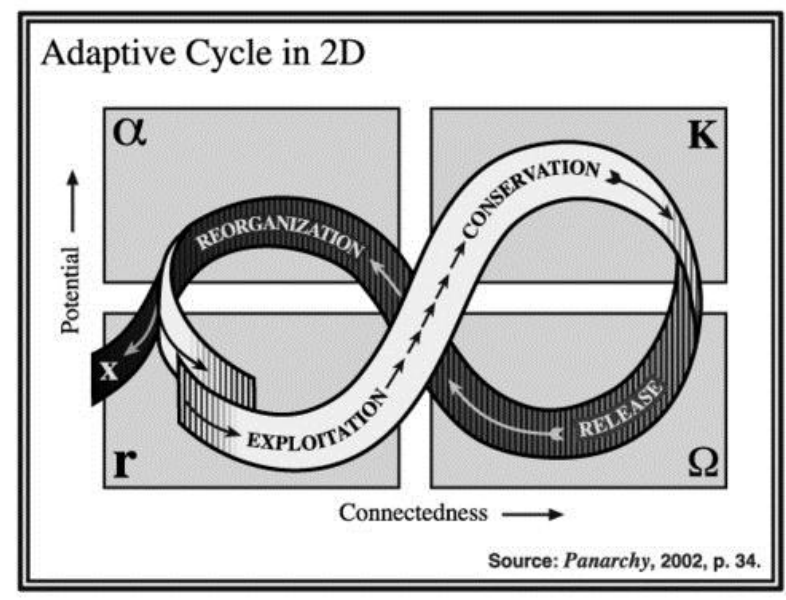

Fig. 1: representação do Ciclo Adaptativo (extraída de Panarchy, 2002, p. 34), que ilustra seus dois momentos principais

\section{O papel da aprendizagem na construção da capacidade adaptativa de SSE}

Repensar os conceitos de sustentabilidade e desenvolvimento à luz das metáforas da resiliência e do ciclo adaptativo nos remete a exemplos tanto de inteiras civilizações que se extinguiram, devido à incapacidade de reorganizar-se em consequência de perturbações, como de comunidades que arregaçaram as mangas para reconstruir as bases da própria sobrevivência e identidade (Diamond, 2006; Tidball e Krasny, 2007). Um destes casos mais recentes e representativos é o da cidade de Detroit, cujos habitantes - envolvidos em um movimento que revolucionou, entre outras coisas, a paisagem urbana - cunharam o slogan "We are turning Motown into Growtown" (estamos transformando a Cidade do Motor na Cidade da (ultivação), para simbolizar a mudança cultural pela qual a cidade passou nos últimos anos (Harris, 2010). A migração da indústria automobilística, da qual Detroit foi o berço nos EUA, para situações geográficas mais lucrativas, deixou atrás de si o desemprego, a pobreza e uma paisagem devastada. A urgência de encontrar saídas da crise levou a

estimulam a criatividade quando aplicadas a novas situações. Desta forma, representam ferramentas valiosas para criar novas ideias e sínteses, que podem sugerir como aplicar uma ideia ou abordagem desenvolvida em determinado domínio a outro completamente diferente (Pickett et al., 2004). 
comunidade urbana a se reorganizar em torno de um projeto que promete reerguer a economia da cidade e melhorar a qualidade de vida de seus habitantes; a solução encontrada consiste em cultivar o solo urbano. Neste contexto, a horticultura urbana não representa apenas um hobby ou uma ocupação extra, mas um componente essencial para a reorganização de todo um sistema. Neste percurso, a comunidade teve de engajar-se em processos de aprendizagem que the permitira abrir mão de valores inspirados na modernidade para resgatar suas raízes e saberes pré-industriais; mas também assimilar e aplicar os saberes científicos e técnicos necessários para resolver problemas associados, por exemplo, à recuperação de solos contaminados e estéreis. Esse processo de aprendizagem colaborativa é amplamente apoiado e promovido por uma rede de organizações governamentais e não governamentais que oferecem todo tipo de recursos para a comunidade local e suas instituições, inclusive educativas ${ }^{6}$.

Exxitos como este reforçam a insuficiência de abordagens - que ainda desfrutam de uma posição privilegiada nas sociedades modernas - fortemente centradas no desenvolvimento e aplicação de conhecimentos científicos especializados, e demonstram, ao mesmo tempo, a efetividade de novas propostas pautadas em uma visão holística ou integrada e, sobretudo, na coprodução de conhecimentos (Lundholm e Plummer, 2010), indispensáveis para projetar (e negociar) cenários alternativos e sustentáveis, e construir a capacidade adaptativa de comunidades.

Essa necessidade abre novas perspectivas de pesquisa e atuação no campo da Educação Ambiental (EA), que, particularmente em consequência da inauguração da Década da Educação para o Desenvolvimento Sustentável, em 2005, vem sofrendo certa polarização. De um lado, posicionam-se aqueles educadores que entendem a EA como meio ou instrumento para se atingir um fim, a sustentabilidade, entendida como conceito não problemático e indisputado, e para os quais a conscientização e o conhecimento preparariam o terreno para mudanças comportamentais e ações voltadas à proteção ambiental. Para seus críticos ${ }^{7}$, esta abordagem tende a engendrar metodologias transmissivas, com ênfase em conteúdos pretensamente universais, na informação e comunicação. Consequentemente, as ações por ela inspiradas padeceriam de certa fixidez, principalmente por não derivarem de decisões participadas, negociadas e tomadas com base em contextos locais. Estes se caracterizam por atributos socioecológicos próprios, e por disputas ideológicas e políticas - visões e projetos de vida, desenvolvimento, etc. travadas nas mais diversas escalas e instâncias, cujo êxito decide as condições de vida e o futuro das comunidades (aqui entendidas em seu sentido mais abrangente, ou seja, compreensivas de todos os seres vivos que habitam certo território).

De outro lado, propõe-se um modelo pautado no valor intrínseco da educação, na qualidade da experiência da aprendizagem e na importância do conhecimento contextualizado. O que importa é cultivar a atitude reflexiva e crítica do sujeito da aprendizagem, para que possa mover-se no campo vasto e disputado dos sentidos da sustentabilidade, fazer escolhas e tomar decisões de maneira autônoma. Entende-se que tais competências sejam imprescindíveis para tomar parte da vida pública, como cidadãos, eleitores e consumidores. Por sua vez, os críticos desta abordagem (de matriz construtivista)

\footnotetext{
${ }^{6}$ http://detroitagriculture.net/

${ }^{7}$ Ver, por exemplo, o Manifesto pela Educação Ambiental redigido por educadores brasileiros e dirigido ao representante da UNESCO no Brasil em ocasião da inauguração da Década da Educação para o Desenvolvimento Sustentável, em 2005. Disponível em: http://www.mma.gov.br/port/sdi/ea/deds/arqs/manifesto_ea.pdf.
} 
argumentam que para se chegar à sustentabilidade é preciso traçar um caminho, planejar estratégias, e que, portanto, seria contraproducente negar toda e qualquer finalidade instrumental da educação.

Mesmo que se admita a complementaridade das duas vertentes sumariamente descritas (Sterling, 2010; Lundholm e Plummer, 2010), resta o fato de que ainda não garantem a produção de uma das condições necessárias para a construção de sistemas socioecológicos sustentáveis e resilientes, qual sejam, a capacidade adaptativa associada à manutenção de suas funções cruciais, tais como a produtividade primária, os ciclos hidrológicos, relações sociais équas, governança e a aprendizagem contínua (Krasny e Roth, 2010). Para estes últimos autores, a capacidade adaptativa se constrói tanto na esfera do indivíduo como na esfera das organizações ou coletivos. No primeiro caso, é necessário que o sujeito se abra a novas maneiras de pensar e pondere suas interpretações, o que poderia ser conseguido mediante sua participação refletida de uma variedade de experiências. 0 segundo caso implica o engajamento em comunidades de prática (Wenger, 2006) voltadas a promover as condições ou atributos de sistemas resilientes, tais como a diversidade cultural e biológica, o capital social, as funções dos ecossistemas, entre outros ${ }^{8}$.

Entre as teorias que melhor propiciam a compreensão e o planejamento de processos de aprendizagem individual e coletiva, a "teoria da aprendizagem situada" e a "teoria da atividade" parecem ser as mais promissoras (Krasny e Roth, op. cit.). A primeira valoriza o contexto da aprendizagem e descreve a maneira pela qual os indivíduos aprendem com as interações sociais e o envolvimento em práticas específicas ${ }^{9}$, ou seja, como interpretam e constroem o mundo mediante essas práticas. Já na teoria da atividade, a ênfase recai na natureza instrumental da atividade e na transformação do ambiente físico com vistas a determinados objetivos.

Esta teoria - que deve sua origem ao trabalho de Vygotsky sobre a importância dos artefatos culturais na mediação de processos de aprendizagem e passou a ser conhecida no mundo ocidental por meio dos estudos desenvolvidos pela escola escandinava a partir dos anos 1980 - considera as mudanças que ocorrem em "sistemas de atividade", tanto nos indivíduos como no contexto, e sugere que a aprendizagem, sempre voltada para finalidades determinadas, se dê na interação dos indivíduos com outros componentes do sistema, como as ferramentas das quais dispõem e os sujeitos com os quais interagem e dividem as tarefas.

\footnotetext{
${ }^{8}$ No campo do planejamento ambiental, esta última abordagem passou a ser conhecida como "aprendizagem social", por resultar da participação em processos colaborativos de gestão dos recursos.

${ }^{9}$ São exemplo de práticas a recuperação de bacias hidrográficas, a jardinagem comunitária, o reflorestamento de áreas degradadas, etc.
} 


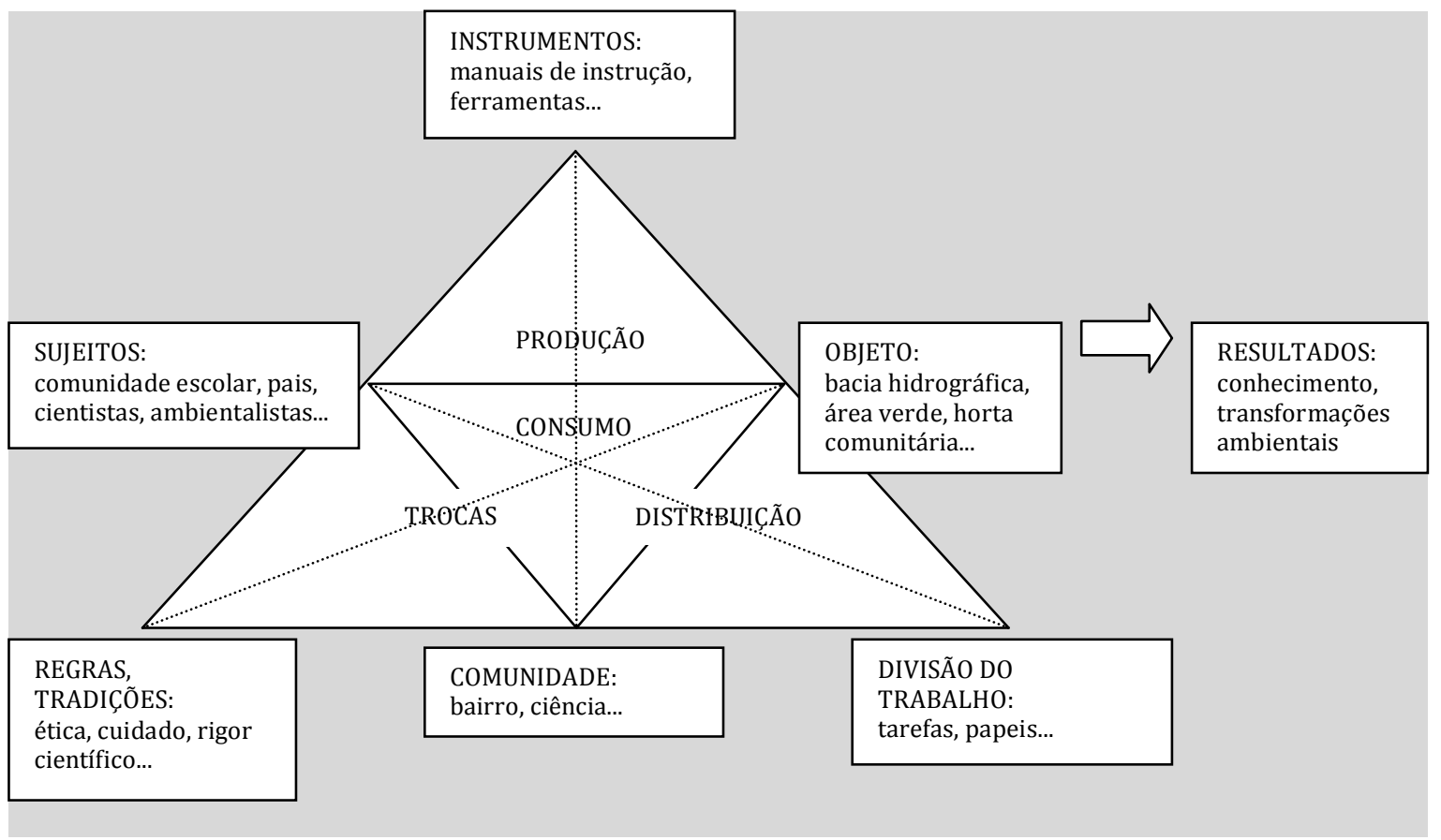

Fig. 2: representação de um Sistema de Atividade (adaptada a partir de Krasny e Roth, 2010)

Em sua história e genealogia da Teoria da Atividade, Engeström (2001) destaca pelo menos cinco princípios nos quais ela pode ser resumida em sua versão mais atual. 0 primeiro princípio considera o sistema de atividade, em suas relações com outros sistemas, como unidade mínima de análise. Isto significa que as ações tanto individuais como coletivas, embora sejam relativamente independentes, podem ser compreendidas somente quando interpretadas adotando-se como pano de fundo inteiros sistemas de atividade. 0 segundo princípio consiste na pluralidade das vozes que se confrontam em sistemas de atividades. Um sistema de atividade configura-se sempre como uma comunidade de múltiplos pontos de vista, tradições e interesses. Em qualquer atividade, a divisão do trabalho coloca os participantes em posições diferentes, eles levam consigo suas diferentes histórias; o próprio sistema de atividade carrega sua própria história, gravada em seus artefatos, regras e convenções. E a historicidade é justamente o terceiro princípio: sistemas de atividade criam-se e transformam-se em longos períodos de tempo. Seus problemas e potencialidades podem ser compreendidos somente se levarmos em conta sua história. 0 quarto princípio considera o papel central das contradições como motor de mudanças e desenvolvimento. Contudo, neste contexto, as contradições não equivalem a um problema ou um conflito, mas consistem em tensões estruturais historicamente acumuladas dentro ou entre sistemas de atividade. A adoção de novos elementos estranhos ao sistema (por exemplo, uma nova tecnologia) em geral comporta um agravamento das contradições, lá onde algum elemento histórico (por exemplo, as normas ou modalidades de divisão do trabalho) se choca com o novo. Tais contradições geram perturbações e conflitos, mas também tentativas inovadoras para mudar a atividade. O quinto e último princípio proclama a possibilidade de transformações expansivas em sistemas de atividade. Sistemas de atividade percorrem ciclos relativamente longos de transformações qualitativas. Na medida em que as contradições de um sistema de atividade se agravam, alguns indivíduos participantes começam a questionar e a transgredir as normas estabelecidas. Em alguns casos, isto resulta em algum tipo de projeção colaborativa e em um esforço deliberado de mudança coletiva. Uma transformação expansiva realiza-se quando o objeto e o motivo da 
atividade são reconceituados para abraçar-se um horizonte muito mais amplo de possibilidades.

Os cinco princípios anteriormente resumidos nos dão uma ideia de quanto a noção e as situações de aprendizagem previstas pela Teoria da Atividade se distanciem daquelas contidas em teorias de aprendizagem mais convencionais, marcadas pela linearidade, pelas quais indivíduos adquirem conhecimentos e competências "estáveis" e previamente definidos, de maneira que se possa avaliar mudanças correspondentes em seu comportamento. Quando, ao contrário, nas transformações mais importantes em nossas vidas pessoais e nas práticas organizacionais, temos de aprender novas formas de atividade que nem sequer estão lá. Elas são literalmente aprendidas à medida que são criadas.

Assim, a Teoria da Atividade, de maneira análoga à metáfora da resiliência, incorpora as ideias da complexidade, da mudança, da adaptação e da contradição - como um tipo de perturbação que leva à aprendizagem e à inovação - bem como a noção de autoorganização, processo pelo qual propriedades do sistema emergem das atividades de indivíduos antes que de determinações superiores. Estas simetrias indicam que programas e projetos educativos concebidos para envolver os alunos em comunidades de práticas e sistemas de atividades voltados à preservação ou recuperação ambiental em contextos concretos podem representar uma estratégia efetiva para capacitá-los a participar ativamente da construção da adaptabilidade e, portanto, da resiliência e sustentabilidade de sistemas socioecológicos.

\section{Reenquadrando as hortas escolares como laboratórios para aprendizagens múltiplas voltadas à construção de SSE sustentáveis e resilientes}

Para Krasny e Tidball (2009) as hortas comunitárias constituem ambientes heterogêneos que integram recuperação ambiental, ativismo comunitário, expressão cultural e segurança alimentar. Do ponto de vista destes autores, podem representar ainda comunidades de práticas nas quais se desenvolvem múltiplas atividades e competências, propiciando uma oportunidade única para diversos tipos de aprendizagem: de conteúdos científicos, e de competências para participar de práticas culturais, políticas e cívicas.

No ensino de ciências, as hortas comunitárias concebidas como laboratórios de aprendizagens múltiplas, na perspectiva da teoria da atividade, proveem uma alternativa para estratégias pedagógicas e didáticas que entendem a aprendizagem como aquisição de conteúdos (princípios, teorias e modelos) descontextualizados e como um processo apenas "interno" ao indivíduo. A oportunidade de interagir com outros sujeitos da comunidade de prática, constituída não apenas pela comunidade escolar, mas por sujeitos e organizações do bairro e da cidade - pais, agricultores e especialistas em diversas disciplinas - permite que crianças e jovens se familiarizem e cotejem, em sua vivência, diversas tradições de conhecimento. Melhor ainda, no espaço da horta comunitária, essas tradições podem ser "executadas" conjuntamente, e isto aproximaria os sujeitos participantes daquele que Turnbull (apud Gough, 2000) considera o autêntico processo de produção de conhecimentos, inclusive do científico, que é situado e se constitui inicialmente a partir de arranjos específicos de condições locais e valores culturais. 
Além de promover a diversidade cultural, tão importante para a capacidade adaptativa de SSE, esta visão do conhecimento e do processo de sua produção em espaços sociais particulares - como no caso da horticultura comunitária, especialmente quando regida por princípios agroecológicos - abre novas perspectivas para a superação das fronteiras entre as culturas das famílias e da comunidade local e aquela da ciência ocidental e da educação em ciências, bem como para mitigar a sensação de inadequação de crianças e jovens ainda não familiarizados com o raciocínio científico (Krasny e Tidball, 2009).

\section{A contribuição da Agroecologia para o desenvolvimento de competências críticas e transformadoras das relações com a natureza}

A Agroecologia é uma ciência pautada no pensamento complexo, que se vale da integração entre conhecimentos técnico-científicos (oriundos de diversos campos disciplinares) e saberes populares (de agricultores tradicionais, povos indígenas e da floresta). Em stricto sensu, pode ser definida como matriz disciplinar que estuda os sistemas agrícolas desde uma perspectiva ecológica, para a construção de agroecossistemas sustentáveis, ou seja, investiga estratégias para intensificar os processos ecológicos que garantem o manejo sustentável de agroecossistemas resilientes (Caporal et al., 2009). De fato, ao propor alternativas ecológicas (como a estimulação da atividade microbiológica do solo para a produção orgânica de nutrientes, o controle biológico baseado em estudos sobre interações ecológicas e técnicas de compostagem de matéria orgânica) a processos industriais, como a fixação sintética de nitrogênio e a fabricação (e uso) intensiva de insumos e inseticidas, etc., a Agroecologia promove a biodiversidade e aquelas sinergias de elementos e processos naturais e humanos que colaboram para o fortalecimento da capacidade adaptativa dos SSE.

Tendo como premissa fundamental a impossibilidade da separação entre a esfera dos fenômenos biológicos e aquela da cultura e da sociedade, essa ciência desenvolve-se na convergência de um enfoque sistêmico, uma abordagem holística e uma ação dialética transformadora, para engendrar não um único modelo globalizante, mas modelos alternativos de desenvolvimento que garantam a soberania e segurança alimentar. Na perspectiva da Agroecologia não existe, de fato, uma solução única, nem fórmulas prontas; cada sistema deveria pesquisar e implementar suas próprias respostas aos problemas e condiç̧̃es locais.

Considerando a importância da agricultura para a vida humana e a urgência de encontrar saídas para a crise socioambiental, para a qual contribui o modelo agroalimentar dominante por violar os princípios fundamentais do ser humano em sua relação com a natureza e o trabalho, a prática da horticultura agroecológica implica o desenvolvimento de competências científicas, críticas e transformadoras das relações com a natureza, essenciais para a construção de comunidades sustentáveis e resilientes. Ainda do ponto de vista educativo, a mesma prática prevê a articulação entre as diferentes disciplinas acadêmicas e escolares, e entre diversas tradições de conhecimento, contribuindo para a superação de uma aprendizagem descontextualizada e fragmentária, e para despertar os sujeitos da aprendizagem para a própria condição de pertencimento a uma comunidade de seres vivos e ao mundo natural. 


\section{Considerações finais}

Neste ensaio, procuramos reenquadrar a prática da horticultura comunitária tendo como principal marco teórico a teoria da atividade aplicada à construção da capacidade adaptativa e da resiliência de SSE sustentáveis. Confiamos que nossas reflexões sobre conceitos, metáforas e teorias procedentes dos campos disciplinares da (Agro)Ecologia e da Educação aportem contribuições teóricas atuais para se repensar não apenas a importância social e educativa da horticultura comunitária, mas também a participação da educação em ciências e da escola no desenvolvimento de comunidades de prática voltadas à aprendizagem colaborativa.

No laboratório da horta comunitária, é possível (re)significar as ciências e o fazer científico, entre outras tradições de conhecimento; a constante realização de indagações, observações, estudos, ponderações, negociações e deliberações inerente ao processo de implantação e gestão de uma horta comunitária agroecológica permite vivenciar a ciência e compreendê-la como uma linguagem, entre outras, que busca interpretar as relações entre as esferas dos fenômeno humanos e naturais.

Por outro lado, o fortalecimento da capacidade adaptativa de SSE demanda que a comunidade escolar repense sua função na formação de competências científicas, políticas e cívicas necessárias para superar a crise socioambiental, e ofereça oportunidades para a aprendizagem situada e colaborativa - necessária para religar os mundos tradicionalmente separados dos seres humanos e da natureza - sem as quais continuará contribuindo para a perpetuação de um cultura insustentável.

\section{Referências}

BECK, U.; GIDDENS, A.; LASH, S. (Orgs.). Modernização reflexiva: política, tradição e estética na ordem social moderna. São Paulo: Editora da Unesp, 1997.

CAPORAL, F.R.; PAULUS, G.; COSTABEBER, J. A. (Orgs.). Agroecologia, uma ciência do campo da complexidade. Brasília (DF). 2009

DIAMOND, J. Collapse: how societies choose to fail or survive. London: Penguin Books, 2006.

ENGESTRÖM, Y. Expansive Learning at Work: toward an activity theoretical reconceptualization. Journal of Education and Work, v.14, n.1, p. 133-156, 2001. Disponível em:

<http://www.handover.eu/upload/library/jimaw6szeyuluh4tho6oq.pdf>. Acesso em: 21 jul. 2013

GLASER, M., KRAUSE, G., RATTER, B., AND WELP, M. Human-Nature-Interaction in the Anthropocene. Potential of Social-Ecological Systems Analysis. Disponível em <http://www.dghumanoekologie.de/pdf/DGH-Mitteilungen/GAIA200801_77_80.pdf>. Acesso em: 21 jul. 2013.

GOUGH, N. Locating curriculum studies in the global village. Journal of Curriculum Studies, v. 32, n. 2, p. 329-342, 2000.

HOLLING, C.S. E GUNDERSON, L.H. Resilience and Adaptive Cycles. In: GUNDERSON, L.H. E HOLLING, C.S. (Eds.). Panarchy: understanding transformations in human and natural systems. Washington: Island Press, 2002, p. 25-62.

KRASNY, M.E. E TIDBALL, K.G. Community Gardens as context for science, stewardship and civic action learning. Cities and the Environment, v. 2, n.1, 18 pp., 2009. Disponível em: <http://escholarship.bc.edu/cate/vol2/iss1/8>. Acesso em: 21 jul. 2013. 
KRASNY, M.E. E ROTH, W.M. Environmental education for social-ecological system resilience: a perspective from activity theory. Environmental Education Research, v. 16, n. 5-6, p.545-558, 2010

LUNDHOLM, C. E PLUMMER, R. Resilience and learning: a conspectus for environmental education. Environmental Education Research, v. 16, n. 5-6, p.475-491, 2010.

PICKETT, S.T.A.; CADENASSO, M.L.; GROVE, J.M. Resilient cities: meaning, models, and metaphor for integrating the ecological, socio-economic, and planning realms. Landscape and Urban Planning, n. 69, p. 369-384, 2004. Disponível em:< http://www.arch.utah.edu/cgi-bin/wordpress-cmp/wpcontent/uploads/2012/09/Pickett\%20-\%20resilient\%20cities.pdf>. Acesso em: 21 jul. 2013

PLUMMER, R. Social-ecological resilience and environmental education: synopsis, application, implications. Environmental Education Research, v. 16, n. 5-6, p.493-509, 2010.

SACHS, W. No sustainability without development. 1995. Disponível em: $<$ http://www.aislingmagazine.com/aislingmagazine/articles/TAM21/Sustainability.html>. Acesso em: 21 jul. 2013.

SCHEFFER, M.; WESTLEY, F; BROCK, W.A.; HOLMGREN, M. Dynamic interaction of societies and ecosystems - Linking theories from Ecology, Economy and Sociology. In:

GUNDERSON, L.H.; HOLLING, C.S. (Eds.). Panarchy: understanding transformations in human and natural systems. Washington: Island Press, 2002, p.195-239.

STERLING, S. Learning for resilience, or the resilient learner? Toward a necessary reconciliation in a paradigm of sustainable education. Environmental Education Research, v.16, n. 5-6, p.511-528, 2010.

TIDBALL, K.G. E KRASNY, M.E. From risk to resilience: what role for community greening and civic ecology in cities? In: WALS, A.E.J. Social Learning: toward a sustainable world. Wageningen: Wageningen Academic Publishers, 2007, p. 149-164.

WENGER, E. Communities of practice: a brief introduction. 2006. Disponível em: $<$ http://www.ewenger.com/theory/>. Acesso em: 21 jul. 2013.

WESTLEY, F.; CARPENTER, S.R.; BROCK, W.A.; HOLLING, C.S.; GUNDERSON, L.H. Why systems of people and nature are not just social and ecological systems. In: GUNDERSON, L.H. e HOLLING, C.S. (Eds.). Panarchy: understanding transformations in human and natural systems. Washington: Island Press, 2002, p.103-120. 